\title{
Immunomodulatory effects of extract of Ganoderma lucidum basidiocarps cultivated on
}

\section{alternative substrate}

Biljana Božić Nedeljkovićc ${ }^{1,2}$, Jasmina Ćilerdžić ${ }^{2}$, Dragana Zmijanjac ${ }^{2}$, Milan Marković ${ }^{1}$, Tanja Džopalić $^{1,3}$, Saša Vasilijić ${ }^{1,4}$, Mirjana Stajić ${ }^{2}$, Dragana Vučević ${ }^{1,4}$

${ }^{1}$ Institute for Medical Research, Military Medical Academy, Belgrade, Serbia.

${ }^{2}$ University of Belgrade, Faculty of Biology, Serbia.

${ }^{3}$ University of Nis, Faculty of Medicine, Serbia.

${ }^{4}$ Faculty of Medicine of the Military Medical Academy, University of Defence in Belgrade, Serbia.

Corresponding author:

Dr. Biljana Božić Nedeljković, Full Professor of immunology

Institute of Physiology and Biochemistry "Ivan Djaja", Faculty of Biology, University of Belgrade,

Serbia

Studentski trg 16, 11000 Belgrade

Phone/Fax: +381 113032356

E-mails: biljana@bio.bg.ac.rs,najbiljana@yahoo.com 


\begin{abstract}
The aim of the study was to investigate if there are any differences in effects of extracts of commercially (GC) and alternatively (wheat straw) (GA) cultivated Ganoderma lucidum basidiocarps on properties of peritoneal macrophages (PM) and monocyte-derived dendritic cells (MoDCs). GA extract stronger stimulated the metabolic and phagocytic activity of PMs, their adhesion capability and ability to produce ROS and NO compared to GC. Both tested extracts significantly increased allostimulatory and Th1 polarization capacity of simultaneous TLR3 and TLR7-activated MoDCs, but GA extract was more effective. The GA extract increased the production of ROS and NO by TLR4 stimulated PMs and up-regulated the production of certain cytokines as well as allostimulatory and Th1 polarization capacity of MoDCs. The GA extract could be a potent immunostimulatory agent for activation of MoDCs with the simultaneous engagement of TLRs that seems to be a promising strategy for preparation of DC-based anti-tumor vaccines.
\end{abstract}

Keywords: Antigen presenting cell; Ganoderma lucidum; Wheat straw; Immunomodulation. 


\section{Introduction}

Ganoderma lucidum (Curtis) P. Karst., known as Reishi, is a popular medicinal mushroom used in traditional medicine for the prevention and treatment of various pathological conditions. Today, there is evidence that bioactive constituents of this species are responsible for numerous health benefits due to strong immunomodulatory, antitumor, antioxidative, anti-inflammatory, antimicrobial, and many other activities (Lin, Kao et al. 2006, Yuen and Gohel 2008, Joseph, Sabulal et al. 2011, Wasser 2014). Therefore, commercial production of this mushroom is continuously growing and nowadays the emphasis is put on the creation of a system for cheaper, easier, faster and environmentally friendly cultivation of biologically more active fruiting bodies. Thus, fruiting bodies with higher immunostimulatory potential could be applied as natural pharmaceutical agents in immunotherapy of patients suffering from various tumor types (Park, Kuen et al. 2018).

The antigen-presenting cells (APCs) are a common checkpoint for stimulation of the immune system and induction of potent antitumor response in cancer treatment (Martin, Schreiner et al. 2015). They include dendritic cells (DCs), macrophages, and B lymphocytes, which participate in capturing, processing and presenting antigens to T lymphocytes (Constantino, Gomes et al. 2017). Macrophages and DCs are the powerful phagocytic cells, key players in the innate immune system and link between innate and adaptive immunity, which are derived from peripheral blood monocytes and exist in almost all tissues (Clark, Angel et al. 2000, Hirayama and Iida 2017). Activated macrophages produce numerous bioactive compounds, including reactive oxygen species (ROS), nitric oxide (NO), an important mediator of innate immune response on various pathological stages, as well as cytokines, primarily interleukins (IL-1, IL-6), tumor necrosis factor $\alpha$ (TNF- $\alpha$ ), and interferon- $\gamma$ (IFN- $\gamma)$, which are crucial in recruitment and activation of other immune cells and stimulation of adaptive immunity (Hirayama and Iida 2017). In the presence of microbes or inflammatory stimuli, DCs undergo a complex process of maturation that includes up-regulation of 
co-stimulatory molecules, migration to lymph nodes, $\mathrm{T}$ lymphocytes priming and cytokine production (Clark, Angel et al. 2000). These potent APCs express various pattern recognition receptors (PRRs) and in such a way trigger signalling pathways resulting in their phenotypic changes and functional maturation (Martin, Schreiner et al. 2015). Toll-like receptors (TLRs) present an important group of PRRs on the macrophages and DCs surface and crucial factors for recognition of viruses, bacteria, fungi, and parasites, i.e. they play a key role in innate immunity (Kawai and Akira 2009). These authors emphasized that ligation of different TLRs by specific TLR agonists presents a powerful tool for induction of DCs maturation. TLR agonists are used as adjuvants or immune modifiers in DC-based trials of tumor immunotherapy) (Gnjatic, Sawhney et al. 2010). However, since single TLR agonist has relatively limited adjuvant effects on DC phenotype and function, current studies are focused on research of synergy between paired TLR agonists (Zheng, Cohen et al. 2008).

Starting from the fact emphasized by $\mathrm{Pi}$ and colleagues ( $\mathrm{Pi}$, Chu et al. 2014) that polysaccharides of Ganoderma spp. possess strong immunostimulatory activity based on their recognition as foreign molecules by various PRRs on DCs, and consequently, on stimulation of APCs maturation, the aim of this study was defined. In our study, we investigated the potential synergism of extracts of commercially (GC) and alternatively (wheat straw) (GA) cultivated $G$. lucidum basidiocarps with different TLRs on different APCs. Namely, we investigated the immunomodulatory effects of GC and GA extracts on functional properties of peritoneal macrophages stimulated by TLR4 and on functional characteristics of human monocyte-derived dendritic cells stimulated by simultaneous engagement of TLR3 and TLR7.

\section{Material and methods}

\section{Organism and growth conditions}

The culture of Ganoderma lucidum BEOFB 431, isolated from fruiting body collected in Bojčin forest (Belgrade, Serbia), is maintained on Malt agar medium in the culture collection of the 
Institute of Botany, Faculty of Biology University of Belgrade. The fruiting bodies were cultivated on alternative substrate consisted of wheat straw under laboratory conditions. Basidiocarps of a commercial Chinese strain, cultivated on oak sawdust, were purchased at a health food store.

\section{Preparation of the basidiocarp extracts}

The dried and pulverized commercially and alternatively produced G. lucidum basidiocarps ( $2.0 \mathrm{~g})$ were extracted with $60.0 \mathrm{ml}$ of $96 \%$ ethanol by stirring on a magnetic stirrer (150 rpm) for $72 \mathrm{~h}$. The resultant extracts were centrifuged $\left(20^{\circ} \mathrm{C}, 3000 \mathrm{rpm}, 10 \mathrm{~min}\right)$ and supernatants were filtered through Whatman No.4 filter paper, concentrated under reduced pressure in a rotary evaporator (Büchi, Rotavapor R-114, Germany) at $40{ }^{\circ} \mathrm{C}$ to dryness, and dissolved in $5 \%$ dimethyl sulphoxide (DMSO) to an initial concentration of $10.0 \mathrm{mg} / \mathrm{ml}$.

\section{Experimental animals}

All animal experiments were approved by the Ethics Review Committee for Animal Experimentation of Military Medical Academy and Ministry of Agriculture and Environmental Protection of Republic of Serbia (Veterinary Directorate No. 323-07-7363/2014-05/5). Inbred male Albino Oxford rats (AO; Vivarium for Small Experimental Animals, Military Medical Academy, Belgrade) weight about 200-220 g were housed in an air-conditioned room at $25{ }^{\circ} \mathrm{C}$ on a $12 \mathrm{~h}$ light/dark cycle. Animals were provided pelleted food (Veterinary Institute, Subotica) and tap water ad libitum. Sacrifice was done with intravenous injection of Ketamin/Xilazyn in a lethal dose. All procedures were done in accordance with the Guide for the care and use of laboratory animals.

\section{Peritoneal macrophages isolation and experimental design}

The medium used for the cell isolation and incubation was HEPES-buffered Roswell Park Memorial Institute medium (RPMI-1640) supplemented with fetal calf serum (FCS) (Flow, Irvine, Ca, USA), glutamine (ICN Flow, SAD), penicillin, and gentamicin (Galenika a.d.d., Serbia). 
Peritoneal cells were obtained by sterile lavage with RPMI medium supplemented with $2 \%$ FCS and heparin (Galenika a.d.d., Serbia). Enrichment of peritoneal cell exudates with PMs was enabled using density gradient OPTIPREP (Nycomed Pharmas, Norway) with $0.8 \% \mathrm{NaCl}$. After centrifugation on the gradient, mononuclear cells (highly enriched with PM, >90\%) were washed and resuspended in RPMI-1640 supplemented with $10 \%$ FCS and cell number was adjusted to $10^{6}$ cells/ml. Afterwards, the cells were seeded in 96-well plate in two ways: $i$ ) $1 \times 10^{5}$ cells per well for testing the viability and production of phagocytic activity, ROS, NO, and cytokine and ii) $5 \times 10^{5}$ cells per well for assessment of adhesion capacity.

Peritoneal macrophages (PMs) isolated in this way were cultivated under standard conditions $\left(37^{\circ} \mathrm{C}, 5 \% \mathrm{CO}_{2}\right)$ for $24 \mathrm{~h}$, and treated with $\mathrm{GC}$ and GA extracts in final concentration of $100.0,10.0$, and $0.1 \mu \mathrm{g} / \mathrm{ml}$ per well in presence or absence of adequate stimulator (depending of evaluated function). Lipopolysaccharide (LPS, Sigma, USA), TLR4 agonist, at a final concentration of $100.0 \mathrm{ng} / \mathrm{ml}$ per well, was used as a stimulator for assessment of metabolic viability, phagocytic activity, and NO production. Adhesion capacity and ROS production were assessed by phorbolmyristate-acetate (PMA, Sigma, USA) at the final concentration of $250.0 \mathrm{ng} / \mathrm{ml}$ per well. Control cells were cultivated under standard conditions, with or without TLR4 agonist and were not treated with GC and GA extracts. All studied functions of PMs were observed after 24 h cultivation in vitro and were done in quadruplicate.

\section{Cell viability assay}

Cell viability was estimated by a quantitative colorimetric assay described for human granulocytes which were based on metabolic reduction of 3-(4,5-dimethylthiazol-2-yl)-2,5diphenyltetrazolium bromide (MTT, Invitrogen) into coloured product formazan (Oez, Platzer et al. 1990). MTT assay was conducted with $24 \mathrm{~h}$ cultivated PMs and MTT which was added in the concentration of $5.0 \mathrm{mg} / \mathrm{ml}\left(10.0 \mu \mathrm{l}\right.$ per well), which were incubated at $37{ }^{\circ} \mathrm{C}$ in an atmosphere of $5 \% \mathrm{CO}_{2}$ and $95 \%$ humidity for $3 \mathrm{~h}$. The absorbance of produced formazan after overnight 
incubation in the solution composed of sodium dodecyl sulphate (SDS) and $\mathrm{HCl}$ (10\% SDS with $0.01 \mathrm{~N} \mathrm{HCl}$ ) was measured at dual wavelengths, $570 / 650 \mathrm{~nm}$ by an ELISA 96-well plate reader (Behringer, Germany). Cells viability was expressed as absorbance of solubilized formazan at the end of the incubation period. The presented data are expressed as the mean \pm standard error (SE) from three independent experiments performed in triplicate.

\section{Phagocytosis assay}

The phagocytic capacity of PMs was determined according to the technique described by Chen and colleagues (Chen, Weng et al. 2015). After $24 \mathrm{~h}$ cultivation PMs without/with stimulators and GC/GA extract, the supernatants were collected and $50 \mu \mathrm{l} /$ well of neutral red (1:300) was added and incubated for $4 \mathrm{~h}$. After incubation, supernatants were discarded, cells were washed with phosphate-buffered saline (PBS) three times and lysed by adding $100.0 \mu \mathrm{l} /$ well of cell lysing solution (ethanol and $1 \%$ acetic acid at the ratio of 1:1), and absorbance of the solution was measured at 540/650 nm using Microplate Reader (Behringer, Germany). The presented results are expressed as the mean \pm SE from three independent experiments performed in triplicate.

\section{Adhesion capacity assay}

Adhesion capacity of PMs was assessed by a method of Oez and colleagues (Oez, Welte et al. 1990) based on the cell ability to adhere to the plastic matrix. After the $24 \mathrm{~h}$ cultivation without/with stimulator (PMA) and GC/GA extract, supernatants were removed and cells were washed three times with warm PBS in order to remove non-adhered cells. Then in each well added methanol $(100.0 \mu \mathrm{l} /$ well $)$ and it was incubated for seven minutes. Attached cells were dyed with $0.1 \%$ solution of methyl blue $(100.0 \mu \mathrm{l} /$ well $)$ for 15 minutes and washed three times with tap water. Plates were left to dry on air overnight and colour was dissolved by adding $0.1 \mathrm{~N} \mathrm{HCl}(200.0$ $\mu 1 /$ well). The absorbance of the solution in each well was measured at 650/570 nm using Microplate 
Reader (Behringer, Germany). The presented results are expressed as the mean \pm SE from three independent experiments performed in triplicate.

\section{NBT reduction assay}

NBT assay was used to evaluate the generation of superoxide anion $\left(\mathrm{O}_{2}^{-}\right)$produced by PMs (Pick, Charon et al. 1981). Briefly, nitroblue tetrazolium (NBT, Invitrogen), in final concentration of $0.5 \mathrm{mg} / \mathrm{ml}$ per well, was added to PMs suspension after $24 \mathrm{~h}$ treatment of PMs without/with stimulators and GC/GA extracts and the mixture was incubated at $37{ }^{\circ} \mathrm{C}$ in an atmosphere of $5 \%$ $\mathrm{CO}_{2}$ and $95 \%$ humidity for one hour. Formed diformazan crystals were dissolved by adding SDS$\mathrm{HCl}$ mixture $(100.0 \mu \mathrm{l} /$ well $)$ and optical density was measured at $570 / 650 \mathrm{~nm}$ by a Microplate reader (Behringer, Germany). The presented results are expressed as the mean \pm SE from three independent experiments performed in triplicate.

\section{Determination of $N O$ production}

Production of NO was quantified by the accumulation of nitrite as a stable end-product and determined by a Greiss reaction assay (Green, Wagner et al. 1982). Equal volumes of the supernatants and Griess reagent [0.35\% 4-aminophenyl sulfone (Sigma-Aldrich, Germany), 0.1\% $\mathrm{N}$-(1-naphthyl)ethylenediamine dihydrochloride in $1 \mathrm{M} \mathrm{HCl}$ (POCh, Poland)] were incubated at room temperature $\left(22 \pm 2{ }^{\circ} \mathrm{C}\right)$ for 10 minutes. The optical density of the solution was measured at 540/650 nm using Microplate Reader (Behringer, Germany). The nitrite concentration $(\mu \mathrm{M})$ was calculated from the prepared standard curve for the known $\mathrm{NaNO}_{2}$ concentrations. The presented results are expressed as the mean \pm SE from three independent experiments performed in triplicate.

\section{Preparation and treatment of human monocyte-derived dendritic cells}

Immature monocyte-derived dendritic cells (MoDCs) were generated from the adherent fraction of human peripheral blood mononuclear cells (PBMCs). Namely, PBMCs from buffy coats 
of healthy volunteers (upon written informed consent) were isolated by density centrifugation in Lymphoprep (Nycomed, Oslo, Norway), re-suspended in $5.0 \mathrm{ml}$ of $10 \%$ FCS with 2Mercaptoethanol (2-ME) in RPMI medium and allowed to adhere to plastic flasks. After incubation at $37^{\circ} \mathrm{C}$ for 90 minutes, non-adhered cells were removed and adhered cells were cultured in $5.0 \mathrm{ml}$ of RPMI medium containing granulocyte-macrophage colony-stimulating factor (GM-CSF; 100.0 $\mathrm{ng} / \mathrm{ml})$ and IL-4 (20.0 ng/ml). On day three, half of medium volume was removed and replaced with the same volume of fresh medium containing GM-CSF and IL-4 and it was incubated for next two days. At the end of the incubation period (on day five), MoDCs $\left(5 \times 10^{5}\right.$ cells/well) were moved in 24-well plate, in RPMI medium containing GM-CSF/IL-4. After six days, immature MoDCs were replated $\left(5 \times 10^{5}\right.$ cells $\left./ \mathrm{ml}\right)$ in medium with different combination of TLR3 agonist (Poly (I:C), Sigma-Aldrich, Germany, $10.0 \mu \mathrm{g} / \mathrm{ml}$ ) and TLR7 agonist (Loxoribine Sigma-Aldrich, Germany, $34.0 \mu \mathrm{g} / \mathrm{ml})$ with GC and GA extracts $(100.0 \mu \mathrm{g} / \mathrm{ml})$, and incubated for $24 \mathrm{~h}$. Afterwards, cell-free supernatants were collected for cytokine analysis, while cells were detached and their phenotype was observed. Cell-free supernatants were collected and stored at $-20{ }^{\circ} \mathrm{C}$ for the subsequent determination of cytokine levels. The cells were used for further studies.

\section{Flow cytometry analysis of MoDCs for immunophenotyping}

MoDC were obtained by cultivation of human monocytes for six days with GM-CSF (100.0 $\mathrm{ng} / \mathrm{ml})$ and IL-4 (20.0 ng/ml). After this period MoDC additionally cultivated in presence of Poly (I:C) (TLR3 agonist) $(10.0 \mu \mathrm{g} / \mathrm{ml})$ and Loxoribine (TLR7 agonist) $(34.0 \mu \mathrm{g} / \mathrm{ml})$ and GC or GA extracts $(100.0 \mu \mathrm{g} / \mathrm{ml})$. Control (non-treated) and those treated MoDCs $\left(2 \times 10^{5}\right.$ cells/tube) were washed in PBS supplemented with $2 \%$ FCS and $0.1 \% \mathrm{NaN}_{3}$, and incubated at $4{ }^{\circ} \mathrm{C}$ for 45 min with one of the following monoclonal antibodies (mAbs): HLA-DR coupled with phycoerythrin (PE), CD83 conjugated with fluorescein isothyocianate (FITC), CD86-PE, CD40-FITC, CD54-PE (Serotec, Oxford, UK), and CCR7-FITC (R\&D Systems, Minneapolis, MN, USA). The control MoDCs incubated with irrelevant mouse Mab reactive with rat antigens were used as the irrelevant 
control. The cells were incubated at $4{ }^{\circ} \mathrm{C}$ for $45 \mathrm{~min}$, washed and fixed with $1 \%$ paraformaldehyde. For cell fluorescence analysis, at least $5 \times 10^{3}$ cells per sample were analysed using EPICS XL-MCL flow cytometer (Coulter, Krefeld, Germany). The cell-surface expression on MoDCs was determined by means of a forward versus side scatter gate.

\section{Mixed leukocyte reaction with allogeneic $\mathrm{CD}^{+}$T lymphocytes}

Conventional $\mathrm{CD}^{+} \mathrm{T}$ lymphocytes were isolated from PBMCs using negative immunomagnetic sorting with $\mathrm{CD}^{+} \mathrm{T}$ lymphocytes isolation MACS kit (MyltenyiBiotec, Germany). According to flow cytometry analysis, the purity of isolated CD4 ${ }^{+} \mathrm{T}$ lymphocytes was higher than $95 \%$. Purified allogeneic $\mathrm{CD}^{+} \mathrm{T}$ lymphocytes $\left(10^{5}\right.$ cells/well $)$ were placed in $96-$ well plates and cultivated with MoDCs pre-treated with TLR agonists and G. lucidum extracts in RPMI medium with $10 \%$ FCS. Cell proliferation was detected after five days of cultivation. Cells were pulsed with $\left[3^{\mathrm{H}}\right]$ thymidine $(1.0 \mu \mathrm{Ci} /$ well; Amersham, UK) for the last $18 \mathrm{~h}$ of cultivation, then harvested onto glass fibre filters, and $\left[3^{\mathrm{H}}\right]$ thymidine incorporation into DNA was measured by $\beta$ scintillation counting (LKB-1219; Rackbeta, Finland). Results were expressed as counts per minute (c.p.m.). Values are given as mean \pm standard deviation (SD). The presented results are representative of three independent experiments ( $n=3$ different donors).

\section{Evaluation of cytokine production}

The levels of TNF- $\alpha$, IL-12, IL-6, and IL-10 were measured in the cell-free supernatants of control and treated MoDCs cultures $\left(4 \times 10^{5}\right.$ cells $\left./ \mathrm{ml}\right)$ by ELISA assays (R\&D Systems, Minneapolis, USA). The levels of cytokines produced by CD4 ${ }^{+} \mathrm{T}$ lymphocytes in co-cultures with MoDCs were evaluated in the cell-free supernatants of co-cultures. Briefly, purified allogeneic $\mathrm{CD}^{+} \mathrm{T}$ cells $\left(1 \times 10^{5}\right.$ cells/well $)$ were cultivated with MoDCs $\left(1 \times 10^{4}\right.$ cells/well $)$ pre-treated with TLR agonists and G.lucidum extracts in RPMI medium with 10\% FCS in 96-well plates. Phorbolmyristate-acetate $(20.0 \mathrm{ng} / \mathrm{ml})$ and ionomycin $(500.0 \mathrm{ng} / \mathrm{ml})$ (Merck, Austria) were added to the 
wells after five days. Cells were incubated for an additional $8 \mathrm{~h}$ and then harvested, and cell-free supernatants were collected after centrifugation and stored at $-20{ }^{\circ} \mathrm{C}$ for the subsequent determination of the studied cytokine levels. Values are given as mean \pm SD. The presented results are representative of three independent experiments $(\mathrm{n}=3$ different donors $)$.

\section{Statistical analysis}

Data were analysed for significant differences using Student`s paired $t$-test. A $p$ value less than 0.05 was considered to be statistically significant.

\section{Results}

Effect of Ganoderma lucidum extracts on metabolic activity/viability of PMs

The PMs cultivated with three different concentrations of commercially or alternatively cultivated G. lucidum basidiocarps (GC and GA, respectively) $(0.1 \mu \mathrm{g} / \mathrm{ml}, 10 \mu \mathrm{g} / \mathrm{ml}$, and 100 $\mu \mathrm{g} / \mathrm{ml}$ ) without (non-stimulated) (Fig. 1A) or with LPS (1 $\mu \mathrm{g} / \mathrm{ml})$ (LPS-stimulated) (Fig. 1B) during $24 \mathrm{~h}$.

The GC and GA extracts have no effect on metabolic activity/viability of non-stimulated PMs, except GC extract in the concentration of $100.0 \mu \mathrm{g} / \mathrm{ml}$ which affected inhibitory (Fig. 1A). However, all tested extracts (except GC in a concentration of $100.0 \mu \mathrm{g} / \mathrm{ml}$ ) showed a stimulatory effect on metabolic activity/viability of LPS-stimulated PMs. Furthermore, in comparison with GC, GA has a stronger effect in concentration $10 \mu \mathrm{g} / \mathrm{mL}$ and $100 \mu \mathrm{g} / \mathrm{ml}$ on metabolic activity/viability of LPS-stimulated PMs (Fig. 1B).

Effect of Ganoderma lucidum extracts on adhesive capability and phagocytic activity of PMs

Effect of G. lucidum extracts cultivated on different substrates (commercially and alternatively) on adhesion capacity of non-stimulated and PMA-stimulated PMs are shown in Fig. 2. The PMs cultivated with three different concentrations of GC or GA extracts $(0.1 \mu \mathrm{g} / \mathrm{ml}, 10$ 
$\mu \mathrm{g} / \mathrm{ml}$, and $100 \mu \mathrm{g} / \mathrm{ml}$ ) without (non-stimulated) (Fig. 2A) or with PMA (250 $\mu \mathrm{g} / \mathrm{ml}$ ) (PMAstimulated) (Fig. 2B) during $24 \mathrm{~h}$. Adhesive capability of attached PMs was assessed by dyed with methyl blue as described in the Materials and Methods. Treatment of non-stimulated PMs with GC extract did not modulate their adhesive capability, while treatment with GA extract, in all tested concentrations, enhanced their adhesive function compared to the ability of non-treated and GC treated PMs (Fig. 2A). Comparing with a control, GC extract had no effect on the adhesive capacity of PMA-stimulated PMs, while GA extract (in all tested concentrations) enhanced this ability (Fig. 2B).

The PMs cultivated with three different concentrations of GC or GA extracts $(0.1 \mu \mathrm{g} / \mathrm{ml}, 10$ $\mu \mathrm{g} / \mathrm{ml}$, and $100 \mu \mathrm{g} / \mathrm{ml}$ ) with LPS (1 $\mu \mathrm{g} / \mathrm{ml}$ ) (LPS-stimulated) during $24 \mathrm{~h}$ (Fig. 3). Phagocytotic/pinocityc activity was assessed by Neutral red uptake as described in the Materials and Methods section.

GC and GA extracts showed no significant effect on the phagocytic function of nonstimulated PMs (data not shown). However, in the case of LPS-stimulated PMs, GA extracts (in all tested concentrations) statistically considerable up-regulated this activity in comparison with nontreated PMs and PMs treated with GC extract (Fig. 3).

\section{Effect of Ganoderma lucidum extracts on ROS and NO production by PMs}

Effect of investigated extracts on the ROS production by PMs treated with LPS after $24 \mathrm{~h}$ treatment compared to the control cell (LPS-stimulated) measured by NBT test as described in the Materials and Methods (Fig. 4). The treatment of non-stimulated PMs with GC and GA extracts did not influence their potential of ROS production (data not shown). Comparing with adequate control cells, GC extract had no effect on ROS production by LPS-stimulated PMs, contrary to GA extract which induced a statistically significant increasing of this ability (Fig. 4).

Effect of investigated extracts on the nitrite level in supernatants of PMs treated with/without LPS after $24 \mathrm{~h}$ treatment compared to control cell (non-stimulated or LPS-stimulated) 
measured by Griess test as described in the Materials and Methods (Fig. 5). The treatment of nonstimulated and LPS-stimulated PMs with GC and GA extracts, in all tested concentrations (except treatment of non-stimulated cells with $100.0 \mu \mathrm{g} / \mathrm{ml}$ of GC extract) caused an increase of NO production. Furthermore, GA extract was a statistically stronger inducer of NO production by tested PMs than GC extract (Fig. 5A and 5B).

\section{Effect of Ganoderma lucidum extracts on phenotype and cytokine production by MoDCs}

Phenotype analysis showed that GC and GA extracts did not affect MoDCs phenotype measured by percentage of HLA-DR, CD83, CD86, CD40, and CCR7 positive cells (data not shown). Simultaneous treatment of MoDCs with treatment with Poly (I:C), Loxoribine and GC or GA extract up-regulated mean of fluorescence (MnI) of CD83 and HLA-DR. Additionally, simultaneous treatment of TLR3 and TLR7-stimulated MoDCs with GA extract also affected the expression of co-stimulatory molecules, CD86 and CD40, as well as a chemokine receptor, CCR7 (Fig. 6A).

The additions of GC extract stimulated the production of IL-6 and TNF- $\alpha$, had no effect on the production of IL-12, and inhibited the production of IL-10 by TLR3 and -7 stimulated MoDCs. On the other hand, GA extract inhibited the production of IL-6 and IL-10 and had no effect on TNF- $\alpha$ and IL-12 production by studied MoDCs compared to MoDC treated only with TLR3 and TLR7 antagonist (Fig. 6B).

Effect of Ganoderma lucidum extracts on alostimulatory and Th cells polarization capacity of MoDCs

The ability of TLR3 and TLR7-stimulated MoDCs to induce the proliferation of allogeneic $\mathrm{CD}^{+} \mathrm{T}$ lymphocytes was not changed by the addition of GC extract, while GA extract significantly enhanced this ability (Fig. 7A). MoDCs treated with TLR3 and TLR7 agonists and with GC extract down-regulated production of IFN- $\gamma$ and had no effect on IL-17 production by allogeneic $\mathrm{CD}^{+} \mathrm{T}$ 
lymphocytes compared to the effect of MoDCs treated only with TLR3 and TLR7 agonists. On the other side, GA extract in combination with TLR3 and TLR7 changed the properties of MoDCs and thus stimulated MoDCs up-regulated IFN- $\gamma$ synthesis and had no effect on IL-17 generation by allogeneic $\mathrm{CD}^{+} \mathrm{T}$ lymphocytes in comparison with TLR3 and TLR7-stimulated MoDCs (Fig. 7B). Additionally, GA extract in combination with TLR3 and TLR7 up-regulated IL-17 synthesis by allogeneic $\mathrm{CD}^{+} \mathrm{T}$ lymphocytes in comparison with GC, TLR3 and TLR7-stimulated MoDCs (Fig. 7B).

\section{Discussion}

Cooperation of different PRR signals in APC during the induction of immune responses is an emerging field in innate immune research. Activation of two or more TLRs, or other PRRs at the same time, which mimic the actual situation during host cell-microbe interaction, may lead to synergistic, antagonistic, or additive effects(Makela, Strengell et al. 2009). On the other side, Ganoderma spp. extracts and metabolites (derived from wild and/or traditionally cultivated basidiocarps) express strong immunomodulatory characteristics and present effective modifiers of different biological processes (Lin, Chen et al. 2006, Boh 2013, Shi, Zhang et al. 2013, Liu, Xing et al. 2016, Liu, Xing et al. 2016). According to available literature, there is no knowledge about the immunomodulatory potential of crude extracts of $G$. lucidum basidiocarps cultivated on any alternative and environmentally-friendly substrate. This is the first report on it and our findings showed that crude extract of G. lucidum basidiocarps cultivated on nutritionally poor wheat straw contained moleculeswith higher immunomodulatory potentials that in cooperation with agonists of different TLRs induced significantly better activation of APCs compared to the effect of TLRs alone. Thus, the GA extract expresses better immunostimulatory potential in cooperation with TLR4 agonist in up-regulation of PMs functional characteristics compared to GC extract as well as induction of better allostimulatory and Th1 polarization capacity of MoDCs treated in cooperation with TLR3 and TLR7 antagonists. Therefore, the extract of alternatively obtained basidiocarps 
could be an effective additional agent during simultaneous engagements of different TLRs on APCs for in vitro preparation of APCs as a tool for anti-tumor therapy.

Today, it is well known that fungi possess the potential for production of high diversity low molecular weight products (secondary metabolites) with various biological activities that are mainly important for their interactions with other organisms (Brakhage and Schroeckh 2011). These secondary metabolites are present in a small amount and their composition/combination depends on the type of substrate on which they are cultivated. It may be a reason for different immunomodulatory effects of GC and GA extracts noted in this study. The literature data and data of this study indicate that the study of the in vitro cultivation conditions that could possibly optimize their production seems of major importance. Another very important fact in the recent modern period is the need to find a cost-effective and environmentally friendly alternative substrate for G. lucidum cultivation which classically was grown on sawdust of sheesham, mango, and poplar.

The genus Ganoderma (especially G. lucidum), has been used since ancient times in Eastern traditional medicine. In recent years, the precise effect and their mechanisms were investigated and G. lucidum in modern days is used in the treatment and prevention of various pathological conditions. The numerous studies have already demonstrated that various Ganoderma spp. extracts and metabolites possess the strong immunostimulatory activity and present effective modifiers of some biological response (Lin, Chen et al. 2006, Boh 2013, Shi, Zhang et al. 2013, Liu, Xing et al. 2016, Liu, Xing et al. 2016). Also, Pi et al. (Pi, Chu et al. 2014) and Lin et al. (Lin, Chen et al. 2006) noted remarkable activation of Th1 and Th2 cells and certain cytokines synthesis in mice treated with $G$. tsugae hot water extract and $G$. formosanum polysaccharide fraction. Similarly, $G$. lucidum polysaccharide, besides effective stimulation of Th1/Th2 immune response, caused stronger proliferation of murine macrophages and significantly higher synthesis of NO as well as IFN- $\gamma$, TNF- $\alpha$, IL-4 and IL-6 in comparison with the control group (Shi, Zhang et al. 2013, Liu, Xing et al. 2016, Liu, Xing et al. 2016). 
Regarding the crucial role of macrophages during establishing and maintaining homeostasis and defending against pathogens and transformed cells, these cells are involved in pathogenesis in many diseases (Jung, Kim et al. 2015, Ampem, Azegrouz et al. 2016, Da Silva and Barton 2016). In response to differences in soluble characteristics of a microenvironment and different signals from microorganism-associated molecular patterns, macrophages can polarize into pro-inflammatory, M1, or anti-inflammatory, M2, phenotype (Lawrence and Natoli 2011, Murray and Wynn 2011). In vitro cultivation of $\mathrm{PMs}$ from mice/rats represents an exceptionally powerful technique to investigate macrophage functions in response to different stimuli, resembling as much as possible the conditions observed in various pathophysiological conditions or as potential therapeutically agents. Thus, glucan isolated from G. lucidum spores has also stimulated cytokine production by PMs in culture (Guo, Xie et al. 2009). The considerable immunostimulatory effect was also exhibited by G. atrum polysaccharide as well as its acetylated form, which caused increased viability of T lymphocytes and level of IL-2 and TNF- $\alpha$ in the serum of immunosuppressive mice treated with cyclophosphamide overdoses, by regulation of ROS production and NF- $\kappa \mathrm{B}$ activity (Chen, Zhang et al. 2014, Yu, Nie et al. 2014, Li, Li et al. 2017). In this study, the immunomodulatory potential of GC and GA extracts was evaluated on PMs with or without TLR4 cooperation (LPS). Macrophages stimulated with LPS are termed as classical activation macrophages (M1 macrophages) and they are involved in the inflammation, pathogen clearance, and anti-tumor immunity (Atri and Guerfali 2018, Shapouri-Moghaddam, Mohammadian et al. 2018). Results of this study show that GC extract with LPS as TLR4 agonist expressed potential for stimulation of metabolic activity/viability of PMs and stimulation of NO production by PMs, while do not express the potential for modulation of adhesive and phagocytic potential and ROS production by PMs. On the other hand, GA extract in combination with TLR4 signals induced by LPS induces intensive stimulation of all investigated functions of PMs including metabolic activity, phagocytic capacity, and production of ROS and NO compared to the effect of GC. Additionally, GA increased the adhesive capacity of PMA-stimulated PMs. These results may indicate that GA 
extract with TLR4 agonist induces stronger signalling that is responsible for stimulation of very significant characteristics of M1 macrophages such as their metabolic activity, phagocytic activity, ROS and NO production compared to TLR4 signalling alone. Also, adhesive capacity of PMAstimulated PMs was additionally increased by GA extracts. A few studies demonstrated the mechanism of mushroom polysaccharides action on cytokine production. Namely, Kim et al. (Kim et al. 2012) and Pi et al. (Pi, Chu et al. 2014)showed that augmentation of TNF- $\alpha$ synthesis by macrophages was based on the polysaccharide binding for TLR4 sited on macrophage membrane and it recognition as pathogen-associated molecular patterns. However, detailed analyses demonstrated that induction of mRNA expression in Sarcoma 180-bearing mice is the main mechanism of the polysaccharide action (Zhang, Nie et al. 2014). Since these studies demonstrated that intracellularly generated ROS (as a response to pathological stimuli) affect NF- $\kappa \mathrm{B}$ activation and in such way cytokine production by macrophages, it can be concluded that ethanol extract of alternatively cultivated $G$. lucidum basidiocarps which significantly increased ROS production in PMs, could be a potent immunostimulatory and anti-tumor agent.

Induction of effective adaptive immune responses dependents on signals from innate immunity especially from the level of maturation of DCs and their characteristics (Constantino, Gomes et al. 2017). In our study, the combination of poly I:C, loxoribine and GC/GA extract induced phenotypic maturation of MoDCs as determined by u-regulating the surface molecules, including HLA-DR and CD83. CD83 acts as a key DC maturation marker (Prechtel, Turza et al. 2007). However, the combination of poly I:C, loxoribine and GA extract induced up-regulation of CD86, CD40 and CCR7. CD86 is a main co-stimulatory ligand for T cells, providing the second signal for proliferation and clone expansion of antigen-specific $\mathrm{T}$ cells (Jeannin, Magistrelli et al. 2000). CD40 is also an indicator of the activation state of MoDCs whose up-regulation acts in favour of enhanced $\mathrm{T}$ cell activation. Interaction of this molecule with its ligand (CD40L), expressed by activated $\mathrm{T}$ cells is important for up-regulation of co-stimulatory molecules on DCs and enhanced capacity of DCs to trigger proliferative responses and for the regulation of DC 
functions (Ara, Ahmed et al. 2018). It is in line with our results which showed significantly higher proliferation of CD4 T lymphocytes co-cultivated with MoDC treated with the combination of poly I:C, loxoribine and GA extract. It is important to mention that solely maturation of DCs, expressing high levels of co-stimulatory and maturation markers, is not sufficient for an adequate immune response. Namely, various cytokine production and subsequent $\mathrm{CD}^{+} \mathrm{T}$ lymphocytes polarization by DCs is of great significance in the induction of proper immune response as well (Zobywalski, Javorovic et al. 2007). Poly I:C is known as a potent stimulator of bioactive IL-12 production and subsequent activator of the Th1 immune response (Rouas, Lewalle et al. 2004). Our findings confirm these published results because Poly I:C in combination with loxoribine and GC/GA extract induces the up-regulation of IL-12 level. An important finding of this study was the intensive promotion of Th1 and slightly promotion of Th17 polarizing capability of MoDCs by simultaneous engagement of poly I:C, loxoribine and GA compared to the capability of MoDCs by simultaneous engagement of poly I:C, loxoribine and GC. Regarding IL-10 production, obtained results are also interesting. Namely, IL-10 is an immunoregulatory cytokine, responsible for tolerogenic properties of DCs (Smits, Engering et al. 2005), which participates in balancing of the immune response (Saraiva, Christensen et al. 2009). Production of IL-10 could be relevant as a down-regulator of extensive production of immunostimulatory cytokines, knowing that the balance between stimulatory and inhibitory cytokines is important for critical point during the immune response. The decreased level of IL-10, in co-culture of CD4 ${ }^{+} \mathrm{T}$ lymphocytes and MoDCs, treated simultaneous with poly I:C and loxoribine and GC/GA, could be explained by mutual antagonistic effects of Th1 cells on Th2 cells and Tregs as showed by Glimcher and Murphy (Glimcher and Murphy 2000). Therefore, down-regulation of IL-10 production may serve as an additional mechanism for the promotion of the Th1 immune response.

Bearing in mind the significance of anti-tumor vaccines, this study performed to find the optimal protocol for the development of DCs able to induce an adequate immune response. The results that we obtained in this study suggest that simultaneous TLR3 and TLR7 signaling with 
signaling induced by components of crude extract of G. lucidum cultivated on wheat straw may provide a previously undescribed approach for DC-based vaccine development by using synergistic TLR ligands and G. lucidum extract. Overall, these results point to that natural immunomodulators possible mechanism for enhancement of effects of known TLR agonists.

\section{Conclusions}

The significance of our results is highlighted by the following facts: $(i)$ immunomodulatory activity is known only for wild and traditionally cultivated G. lucidum basidiocarps; (ii) traditional cultivation of G. lucidum basidiocarps on various hardwood sawdusts is not ecologically and economically friendly; (iii) substratecomposition and cultivation conditions significantly affected type and content of bioactive metabolites and their activities (Cilerdzic, Sofrenic et al. 2018); (iv) wheat straw induced a synthesis of numerous bioactive molecules, primarily triterpenoids (Cilerdzic, Sofrenic et al. 2018), which could be considered as the one of strong modulators of APCs activity; $(v)$ there is increasing need for natural immunomodulators, without any side effect; (vi) modern vaccines based on highly purified antigen induce insufficient immune protection and a need for natural vaccine adjuvants is continuously growing.

\section{Acknowledgments}

This work was supported by Ministry of Education, Science and Technological Development of the Republic of Serbia (451-03-68/2020-14/ 200178) and Ministry of Defence of the Republic of Serbia (MFVMA/10/13-15).

\section{Disclosure of interest}

The authors declare that there is no conflict of interest. 


\section{References}

Ampem, G., H. Azegrouz, A. Bacsadi, L. Balogh, S. Schmidt, J. Thuroczy and T. Roszer (2016). "Adipose tissue macrophages in non-rodent mammals: a comparative study." Cell Tissue $\underline{\operatorname{Res} 363(2): ~ 461-478 . ~}$

Ara, A., K. A. Ahmed and J. Xiang (2018). "Multiple effects of CD40-CD40L axis in immunity against infection and cancer." Immunotargets Ther7: 55-61.

Atri, C. and F. Z. Guerfali (2018). "Role of Human Macrophage Polarization in Inflammation during Infectious Diseases." 19(6).

Boh, B. (2013). "Ganoderma lucidum: a potential for biotechnological production of anti-cancer and immunomodulatory drugs." Recent Pat Anticancer Drug Discov8(3): 255-287.

Brakhage, A. A. and V. Schroeckh (2011). "Fungal secondary metabolites - strategies to activate silent gene clusters." Fungal Genet Biol48(1): 15-22.

Chen, H. Y., I. C. Weng, C. S. Li, L. Wan and F. T. Liu (2015). "Examination of galectins in phagocytosis." Methods Mol Biol1207: 201-213.

Chen, Y., H. Zhang, Y. Wang, S. Nie, C. Li and M. Xie (2014). "Acetylation and carboxymethylation of the polysaccharide from Ganoderma atrum and their antioxidant and immunomodulating activities." Food Chem 156: 279-288.

Cilerdzic, J. L., I. V. Sofrenic, V. V. Tesevic, I. D. Brceski, S. N. Duletic-Lausevic, J. B. Vukojevic and M. M. Stajic (2018). "Neuroprotective Potential and Chemical Profile of Alternatively Cultivated Ganoderma lucidum Basidiocarps." Chem Biodivers15(5): e1800036.

Clark, G. J., N. Angel, M. Kato, J. A. Lopez, K. MacDonald, S. Vuckovic and D. N. Hart (2000). "The role of dendritic cells in the innate immune system." Microbes Infect2(3): 257-272.

Constantino, J., C. Gomes, A. Falcao, B. M. Neves and M. T. Cruz (2017). "Dendritic cell-based immunotherapy: a basic review and recent advances." Immunol Res65(4): 798-810.

Da Silva, N. and C. R. Barton (2016). "Macrophages and dendritic cells in the post-testicular environment." Cell Tissue Res363(1): 97-104. 
Glimcher, L. H. and K. M. Murphy (2000). "Lineage commitment in the immune system: the T helper lymphocyte grows up." Genes Dev14(14): 1693-1711.

Gnjatic, S., N. B. Sawhney and N. Bhardwaj (2010). "Toll-like receptor agonists: are they good adjuvants?" Cancer J16(4): 382-391.

Green, L. C., D. A. Wagner, J. Glogowski, P. L. Skipper, J. S. Wishnok and S. R. Tannenbaum (1982). "Analysis of nitrate, nitrite, and [15N]nitrate in biological fluids." Anal Biochem126(1): 131-138.

Guo, L., J. Xie, Y. Ruan, L. Zhou, H. Zhu, X. Yun, Y. Jiang, L. Lu, K. Chen, Z. Min, Y. Wen and J. $\mathrm{Gu}$ (2009). "Characterization and immunostimulatory activity of a polysaccharide from the spores of Ganoderma lucidum." Int Immunopharmacol9(10): 1175-1182.

Hirayama, D. and T. Iida (2017). "The Phagocytic Function of Macrophage-Enforcing Innate Immunity and Tissue Homeostasis." 19(1).

Jeannin, P., G. Magistrelli, J. P. Aubry, G. Caron, J. F. Gauchat, T. Renno, N. Herbault, L. Goetsch, A. Blaecke, P. Y. Dietrich, J. Y. Bonnefoy and Y. Delneste (2000). "Soluble CD86 is a costimulatory molecule for human T lymphocytes." Immunity13(3): 303-312.

Joseph, S., B. Sabulal, V. George, K. R. Antony and K. K. Janardhanan (2011). "Antitumor and anti-inflammatory activities of polysaccharides isolated from Ganoderma lucidum." Acta Pharm61(3): 335-342.

Jung, D. H., K. H. Kim, H. E. Byeon, H. J. Park, B. Park, D. K. Rhee, S. H. Um and S. Pyo (2015). "Involvement of ATF3 in the negative regulation of iNOS expression and NO production in activated macrophages." Immunol Res62(1): 35-45.

Kawai, T. and S. Akira (2009). "The roles of TLRs, RLRs and NLRs in pathogen recognition." Int Immunol21(4): 317-337.

Kim, H. S., Y. J. Kim, H. K. Lee, H. S. Ryu, J. S. Kim, M. J. Yoon, J. S. Kang, J. T. Hong, Y. Kim and S. B. Han (2012). "Activation of macrophages by polysaccharide isolated from Paecilomyces cicadae through toll-like receptor 4." Food Chem Toxicol50(9): 3190-3197. 
Lawrence, T. and G. Natoli (2011). "Transcriptional regulation of macrophage polarization: enabling diversity with identity." Nat Rev Immunol11(11): 750-761.

Li, W. J., L. Li, W. Y. Zhen, L. F. Wang, M. Pan, J. Q. Lv, F. Wang, Y. F. Yao, S. P. Nie and M. Y. Xie (2017). "Ganoderma atrum polysaccharide ameliorates ROS generation and apoptosis in spleen and thymus of immunosuppressed mice." Food Chem Toxicol99: 199-208.

Lin, J. Y., M. L. Chen and B. F. Lin (2006). "Ganoderma tsugae in vivo modulates Th1/Th2 and macrophage responses in an allergic murine model." Food Chem Toxicol44(12): 2025-2032.

Lin, K. I., Y. Y. Kao, H. K. Kuo, W. B. Yang, A. Chou, H. H. Lin, A. L. Yu and C. H. Wong (2006). "Reishi polysaccharides induce immunoglobulin production through the TLR4/TLR2mediated induction of transcription factor Blimp-1." J Biol Chem281(34): 24111-24123.

Liu, Z., J. Xing, Y. Huang, R. Bo, S. Zheng, L. Luo, Y. Niu, Y. Zhang, Y. Hu, J. Liu, Y. Wu and D. Wang (2016). "Activation effect of Ganoderma lucidum polysaccharides liposomes on murine peritoneal macrophages." Int J Biol Macromol82: 973-978.

Liu, Z., J. Xing, S. Zheng, R. Bo, L. Luo, Y. Huang, Y. Niu, Z. Li, D. Wang, Y. Hu, J. Liu and Y. $\mathrm{Wu}$ (2016). "Ganoderma lucidum polysaccharides encapsulated in liposome as an adjuvant to promote Th1-bias immune response." Carbohydr Polym142: 141-148.

Makela, S. M., M. Strengell, T. E. Pietila, P. Osterlund and I. Julkunen (2009). "Multiple signaling pathways contribute to synergistic TLR ligand-dependent cytokine gene expression in human monocyte-derived macrophages and dendritic cells." J Leukoc Biol85(4): 664-672.

Martin, K., J. Schreiner and A. Zippelius (2015). "Modulation of APC Function and Anti-Tumor Immunity by Anti-Cancer Drugs." Front Immunol6: 501.

Murray, P. J. and T. A. Wynn (2011). "Obstacles and opportunities for understanding macrophage polarization." J Leukoc Biol89(4): 557-563.

Oez, S., E. Platzer and K. Welte (1990). "A quantitative colorimetric method to evaluate the functional state of human polymorphonuclear leukocytes." Blut60(2): 97-102.

Oez, S., K. Welte, E. Platzer and J. R. Kalden (1990). "A simple assay for quantifying the inducible adherence of neutrophils." Immunobiology180(4-5): 308-315. 
Park, Y. J., D. S. Kuen and Y. Chung (2018). "Future prospects of immune checkpoint blockade in cancer: from response prediction to overcoming resistance." 50(8): 109.

Pi, C. C., C. L. Chu, C. Y. Lu, Y. J. Zhuang, C. L. Wang, Y. H. Yu, H. Y. Wang, C. C. Lin and C. J. Chen (2014). "Polysaccharides from Ganoderma formosanum function as a Th1 adjuvant and stimulate cytotoxic T cell response in vivo." Vaccine 32(3): 401-408.

Pick, E., J. Charon and D. Mizel (1981). "A rapid densitometric microassay for nitroblue tetrazolium reduction and application of the microassay to macrophages." $\mathrm{J}$ Reticuloendothel Soc30(6): 581-593.

Prechtel, A. T., N. M. Turza, A. A. Theodoridis and A. Steinkasserer (2007). "CD83 knockdown in monocyte-derived dendritic cells by small interfering RNA leads to a diminished $\mathrm{T}$ cell stimulation." J Immunol178(9): 5454-5464.

Rouas, R., P. Lewalle, F. El Ouriaghli, B. Nowak, H. Duvillier and P. Martiat (2004). "Poly(I:C) used for human dendritic cell maturation preserves their ability to secondarily secrete bioactive IL12." Int Immunol16(5): 767-773.

Saraiva, M., J. R. Christensen, M. Veldhoen, T. L. Murphy, K. M. Murphy and A. O'Garra (2009). "Interleukin-10 production by Th1 cells requires interleukin-12-induced STAT4 transcription factor and ERK MAP kinase activation by high antigen dose." Immunity31(2): 209-219.

Shapouri-Moghaddam, A., S. Mohammadian, H. Vazini, M. Taghadosi, S. A. Esmaeili, F. Mardani, B. Seifi, A. Mohammadi, J. T. Afshari and A. Sahebkar (2018). "Macrophage plasticity, polarization, and function in health and disease." 233(9): 6425-6440.

Shi, M., Z. Zhang and Y. Yang (2013). "Antioxidant and immunoregulatory activity of Ganoderma lucidum polysaccharide (GLP)." Carbohydr Polym95(1): 200-206.

Smits, H. H., A. Engering, D. van der Kleij, E. C. de Jong, K. Schipper, T. M. van Capel, B. A. Zaat, M. Yazdanbakhsh, E. A. Wierenga, Y. van Kooyk and M. L. Kapsenberg (2005). "Selective probiotic bacteria induce IL-10-producing regulatory $\mathrm{T}$ cells in vitro by modulating dendritic cell function through dendritic cell-specific intercellular adhesion molecule 3-grabbing nonintegrin." $\underline{\mathbf{J}}$ Allergy Clin Immunol115(6): 1260-1267.

Wasser, S. P. (2014). "Medicinal mushroom science: Current perspectives, advances, evidences, and challenges." Biomed J37(6): 345-356. 
Yu, Q., S. P. Nie, J. Q. Wang, P. F. Yin, D. F. Huang, W. J. Li and M. Y. Xie (2014). "Toll-like receptor 4-mediated ROS signaling pathway involved in Ganoderma atrum polysaccharide-induced tumor necrosis factor-alpha secretion during macrophage activation." Food Chem Toxicol66: 14-22. Yuen, J. W. and M. D. Gohel (2008). "The dual roles of Ganoderma antioxidants on urothelial cell DNA under carcinogenic attack." J Ethnopharmacol118(2): 324-330.

Zhang, S., S. Nie, D. Huang, J. Huang, Y. Feng and M. Xie (2014). "A polysaccharide from Ganoderma atrum inhibits tumor growth by induction of apoptosis and activation of immune response in CT26-bearing mice." J Agric Food Chem62(38): 9296-9304.

Zheng, R., P. A. Cohen, C. A. Paustian, T. D. Johnson, W. T. Lee, S. Shu and G. K. Koski (2008). "Paired Toll-like receptor agonists enhance vaccine therapy through induction of interleukin-12." Cancer Res68(11): 4045-4049.

Zobywalski, A., M. Javorovic, B. Frankenberger, H. Pohla, E. Kremmer, I. Bigalke and D. J. Schendel (2007). "Generation of clinical grade dendritic cells with capacity to produce biologically active IL-12p70." J Transl Med5: 18. 


\section{Figures:}

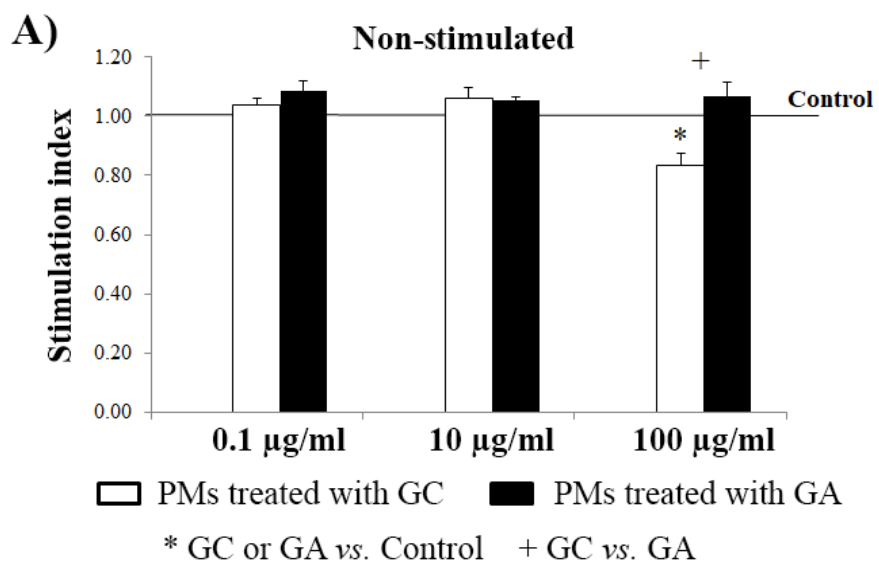

B) LPS-stimulated

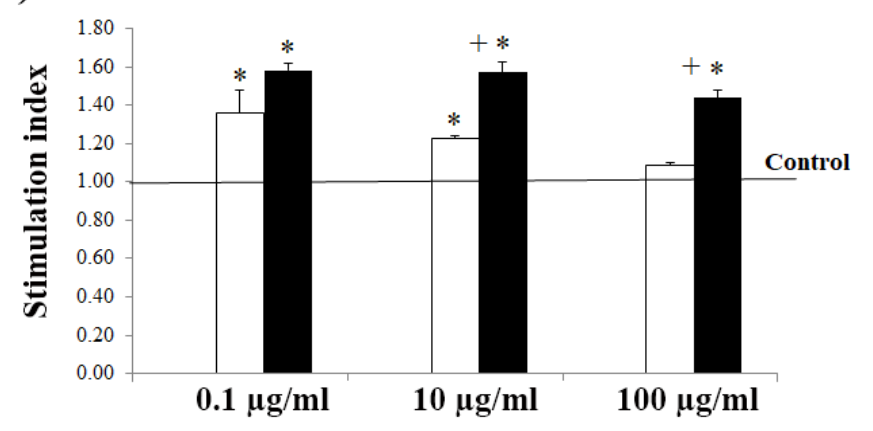

Figure 1. Effect of Ganoderma lucidum extracts on metabolic activity/viability of PMs.

A)

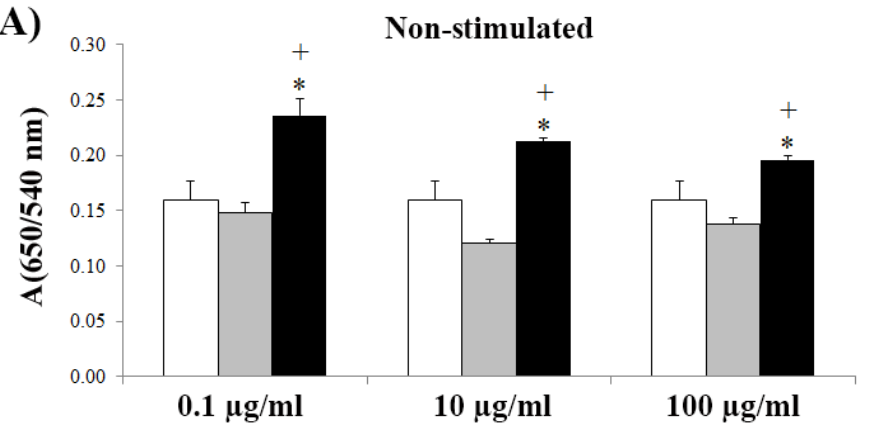

$\square$ Control $\square$ PMs treated with GC

PMs treated with GA

* GC or GA vs. Control + GC vs. GA

B)

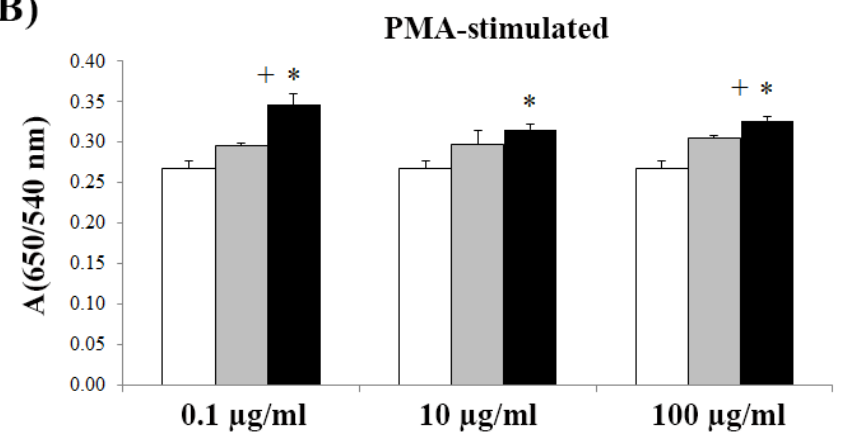

Figure 2. Effect of Ganoderma lucidum extracts on adhesive capability of PMs. 


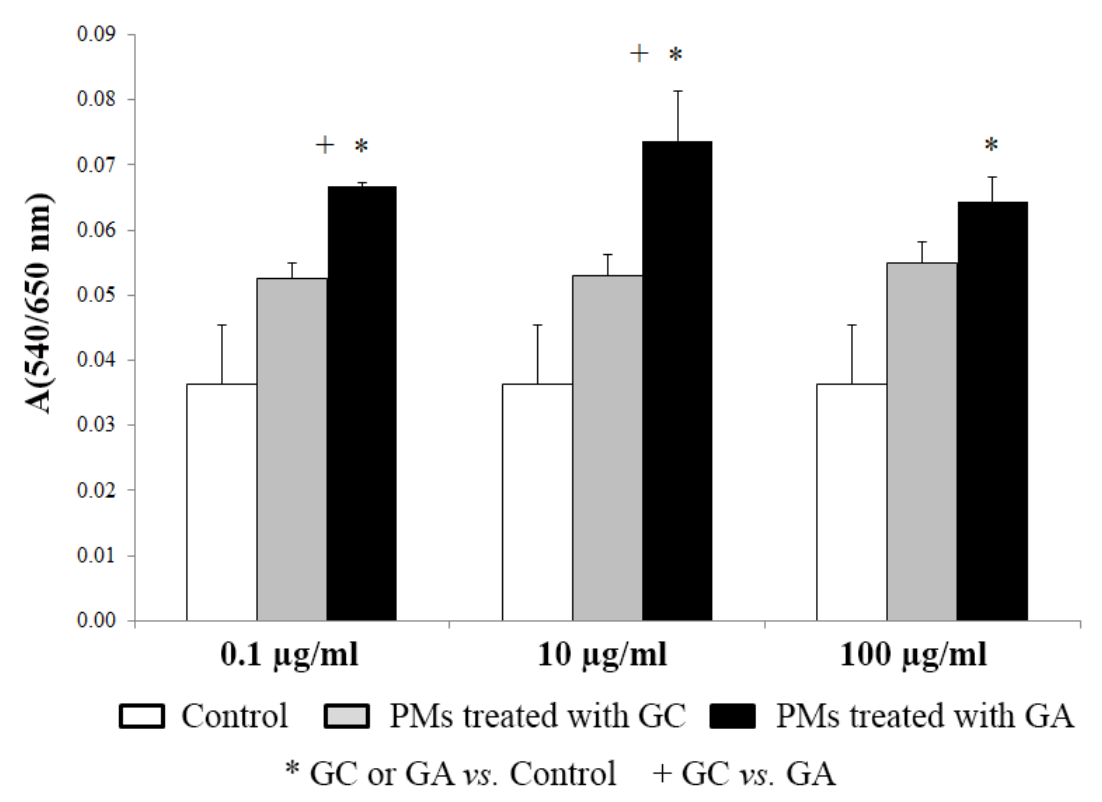

Figure 3. Effect of Ganoderma lucidum extracts on phagocytic activity of LPS stimulated PMs.

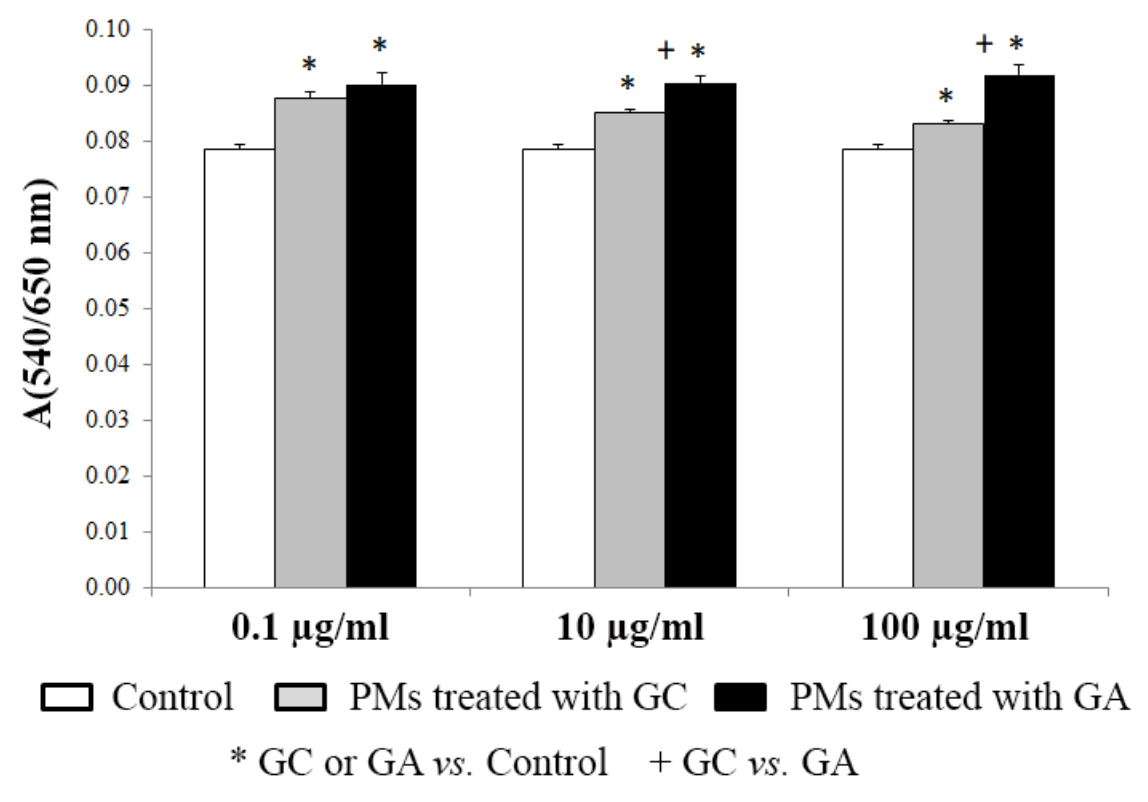

Figure 4. Effect of Ganoderma lucidum extracts on ROS production by LPS stimulated PMs. 
A)

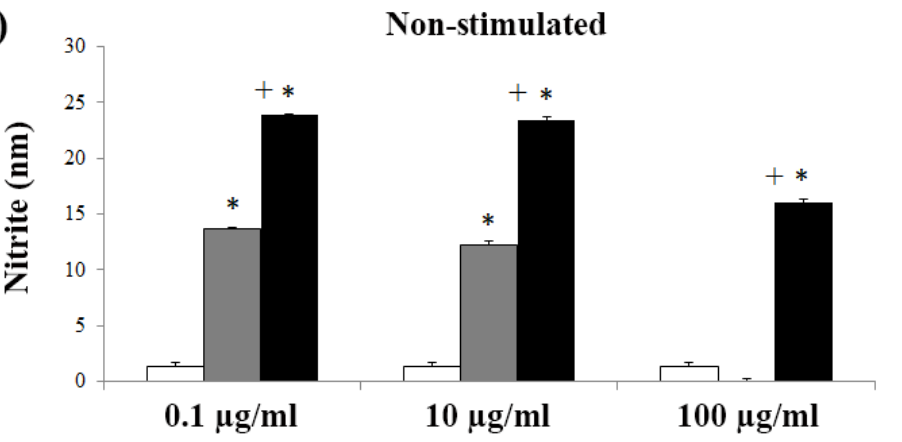

$\square$ Control $\square$ PMs treated with GC

PMs treated with GA

* GC or GA vs. Control + GC vs. GA

B)

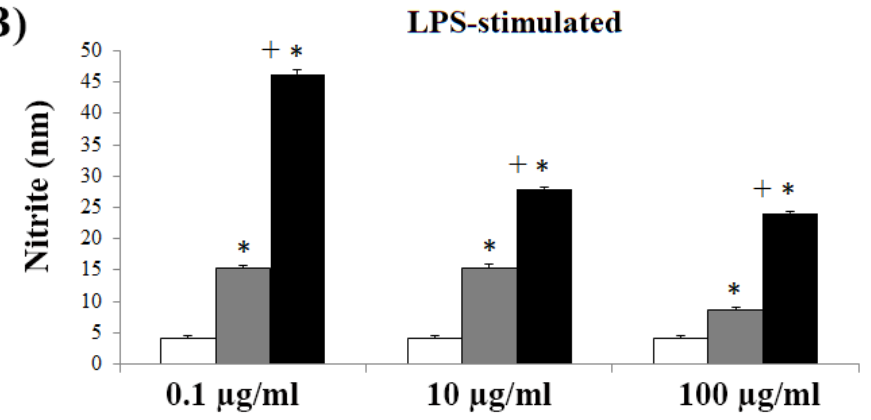

Figure 5. Effect of Ganoderma lucidum extracts on NO production by PMs.
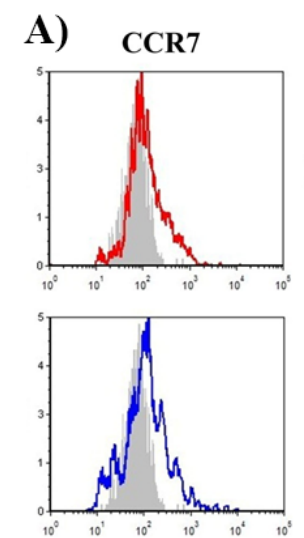

Mean

MoDC TLR3/7

MoDC TLR3/7+ GC 1144.3

MoDC TLR3/7+ GA 1005.8
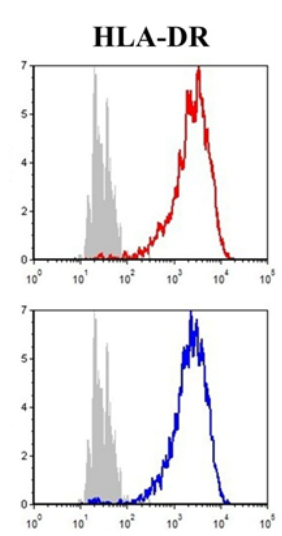

HLA-DR CD83 CD86 CD40 CCR7

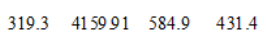

$377.8 \quad 415708$

$384.1 \quad 496626 \quad 682.99 \quad 493.6$
B)

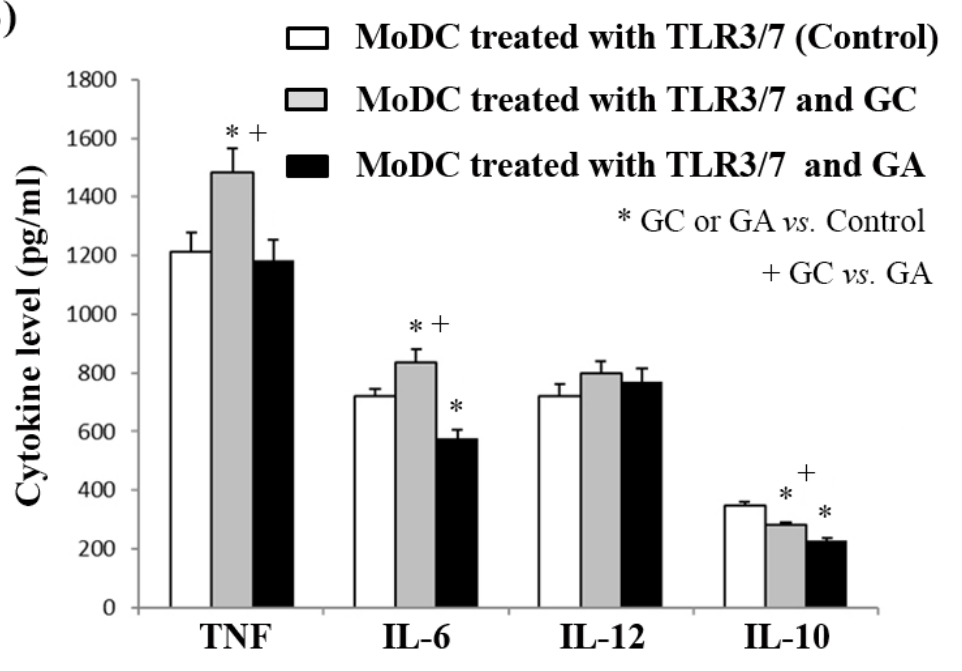

Figure 6. Effect of Ganoderma lucidum extracts on phenotype (The red line represents expression for MoDC TLR3/7 + GC, the blue line represents expression for MoDC TLR3/7 + GA, the full gray histogram represents expression for MoDC TLR3/7) (A) and cytokine production (B) by MoDCs. 

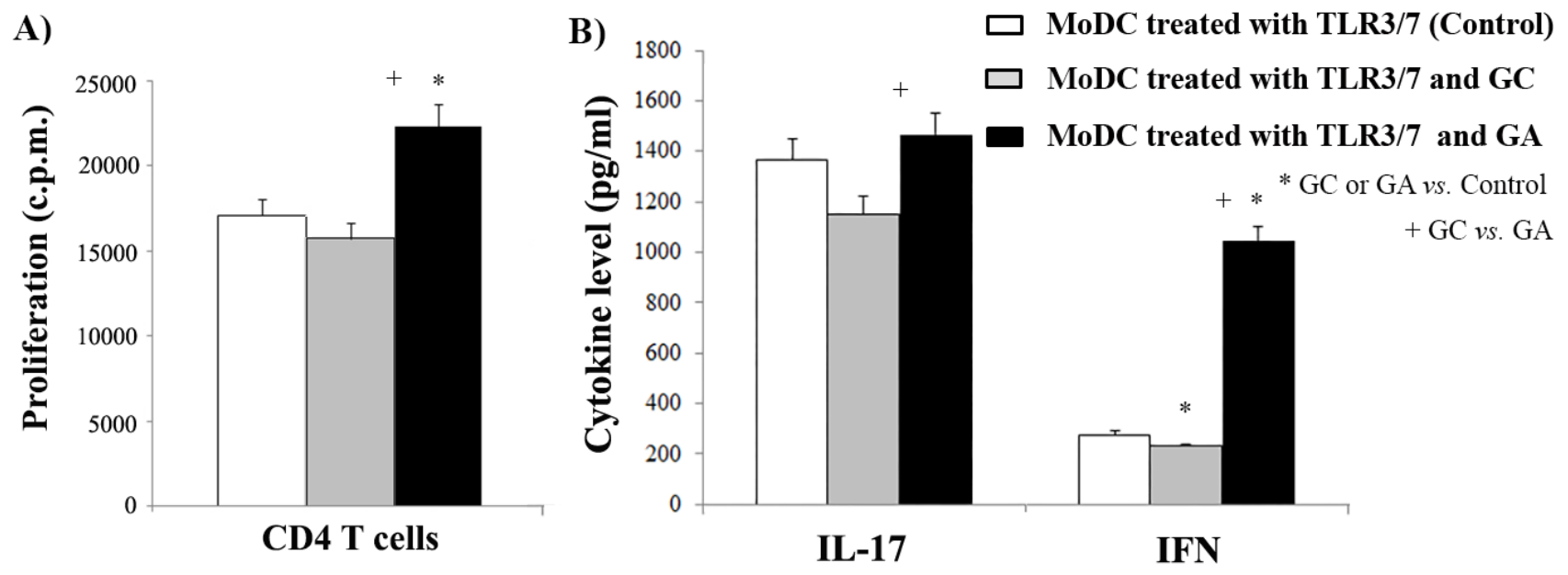

Figure 7. Effect of Ganoderma lucidum extracts on alostimulatory (A) and Th polarization capacity (B) of MoDCs 


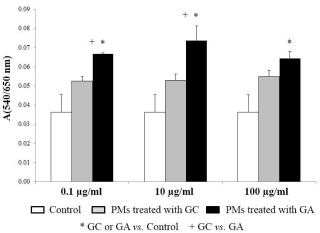




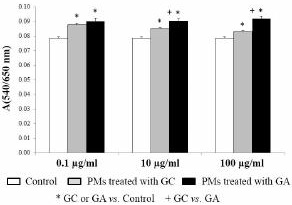




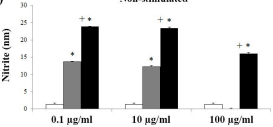

$\square$ Control $\square$ PMs treated with GC $\square$ PMs treated with GA * GC or GA vs. Control + GC vs. GA

B)

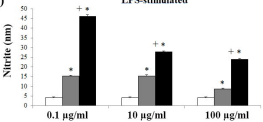


сеит
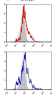

at

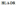

tat

Acer: The

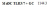

Merctuest+ E6 nes?
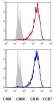

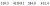

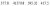

wh ens nes ins
B)

$\square$ MobC treeted wath TI.

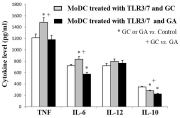


A)

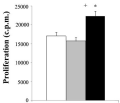

CD4 T eells
B)

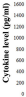
$\square$ MbDC uxated with TI.R3? and C.C

- MoDC ireated with TLR3 and GA

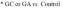

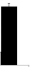

$+K^{*}$ w 4 Sh 
A)

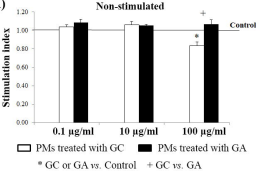

B)

LPS-stimulated

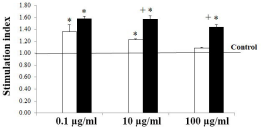


A)

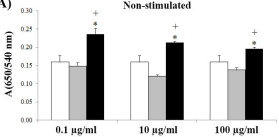

$\square$ Control $\square$ PMs treated with GC $\square$ PMs treated with GA * GC or GA vs. Control + GC ws. GA

B)

\section{PMA-stimulated}

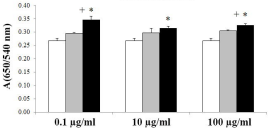

